\title{
Magnetic resonance imaging of the pulmonary arteries and their systemic connections in pulmonary atresia: comparison with angiographic and surgical findings
}

\author{
R S O REES, JANE SOMERVILLE, S R UNDERWOOD, JULIE WRIGHT, \\ D N FIRMIN, R H KLIPSTEIN, D B LONGMORE \\ From the Magnetic Resonance Unit, National Heart and Chest Hospitals, and Paediatric and Adolescent Unit, \\ National Heart Hospital, London
}

SUMMARY Patients with pulmonary atresia require several investigations and operations. The role of magnetic resonance imaging in assessing the anatomy of the central pulmonary arteries, the origin and course of systemic collateral arteries, and the patency of surgical shunts has been studied with the aim of reducing the need for invasive angiography. Transverse, coronal, and sagittal images were obtained in ten adult patients and assessed without knowledge of surgical and angiographic data. Central pulmonary artery anatomy varied from full development to complete absence. Transverse slices showed hypoplastic arteries particularly well and the findings accorded with surgical and angiographic data in all patients. The origin and proximal course of 15 large collaterals were identified on the magnetic resonance images and 18 were identified by surgical and angiographic data. Magnetic resonance imaging did not show their distal connections; if such information is required angiography will be needed. Five surgical shunts were shown to be patent and two occluded at surgery and angiography, and this was confirmed on the magnetic resonance images. The patency of a further four shunts was uncertain, but they were not seen by magnetic resonance and were presumed to be occluded.

Patients with pulmonary atresia depend upon branches of the systemic circulation to provide a pulmonary blood supply. Development of pulmonary arteries centrally and segmentally is variable, as is the size and number of the systemic collateral arteries, and there tends to be an inverse relation between the two. ${ }^{1}$ Successful palliative or radical reparative surgery depends on complete demonstration of both, and currently this information cannot be obtained non-invasively, but requires angiography with multiple contrast injections and selective catheterisation of individual collateral arteries. ${ }^{2-4}$ It is also important to establish the state of surgical shunts. ${ }^{5}$

Requests for reprints to Dr R S O Rees, Magnetic Resonance Unit, National Heart and Chest Hospitals, 30 Britten Street, London SW3 6NN.

Accepted for publication 21 July 1987
The role of magnetic resonance imaging in assessing the anatomy of the central pulmonary arteries, the origin and course of systemic collateral arteries, and the patency of surgical shunts has been studied and compared with angiographic and surgical findings with the ultimate aim of reducing the need for invasive investigation.

\section{Patients and methods}

We studied 10 adult patients (seven men and three women aged 19-40 (mean 24)). We chose to study adults because sedation was not used and it was essential for the patients to lie still during the acquisition of the images. Eight had a subaortic ventricular septal defect with normal atrioventricular connections. Two had abnormal atrioventricular connections. Up to the time of the study a total of 39 angiographic investigations and 13 operations had 
been performed in the 10 patients (table).

The magnetic resonance images were acquired with a Picker International Vista MR2055 machine with a superconducting magnet operating at $0.5 \mathrm{~T}$. A spin echo sequence (echo time $40 \mathrm{~ms}$ ) was used, and the radiofrequency signals were received with specially constructed thoracic surface coils. Images were acquired in transverse, coronal, and sagittal planes through the area of interest. Six or eight $1 \mathrm{~cm}$ thick tomographic slices were obtained simultaneously with electrocardiographic gating. Where indicated these were repeated with an offset of $0.5 \mathrm{~cm}$ to give an interleaved set of $1 \mathrm{~cm}$ thick slices $0.5 \mathrm{~cm}$ apart. The pixel size was $1.2 \mathrm{~mm} \times 1.2 \mathrm{~mm}$ and each group of slices was obtained over $256 \mathrm{car}-$ diac cycles, consisting of two acquisitions for each of 128 phase encoding steps. In order to maximise the contrast between moving blood and soft tissue, data acquisition was concentrated into the systolic period, and by use of the minimum repeat time it was possible to acquire eight slices within $560 \mathrm{~ms}$ of

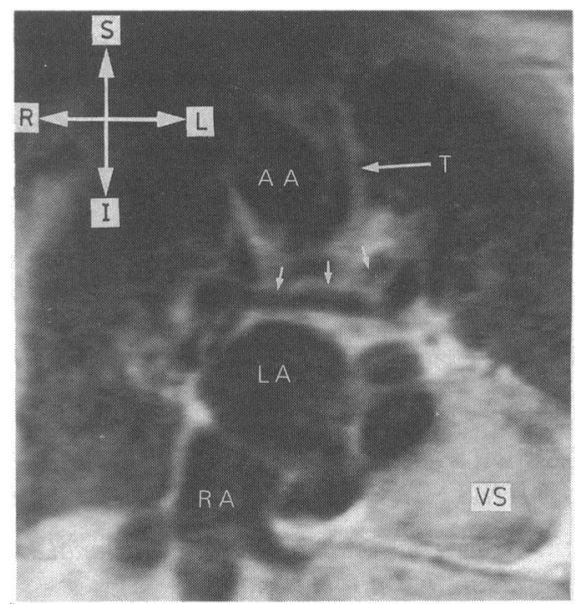

Fig 1 Magnetic resonance image of coronal slice in patient 8 showing hypoplastic central pulmonary arteries (arrows). The aortic arch $(A A)$ is to the right. $L A$, left atrium; $R A$, right atrium; $T$, trachea; $V S$, ventricular septum.

Table Data on patients

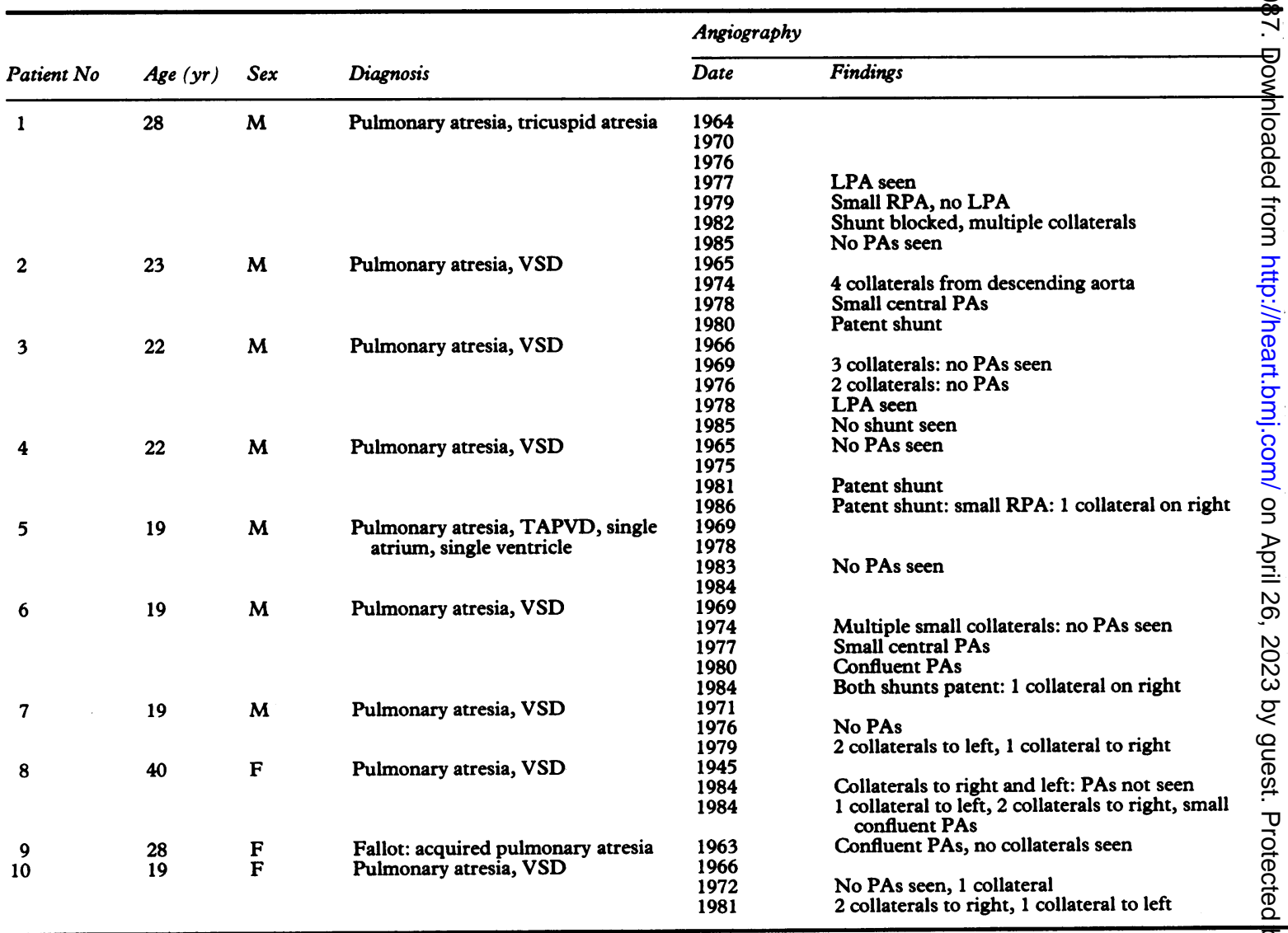

Ao, aorta; collateral, systemic-pulmonary collateral artery; L, left; PA, pulmonary artery; PT, pulmonary trunk; R, right; SA, subclavian artery< TAPVD, total anomalous pulmonary venous drainage; VSD, ventricular septal defect. 


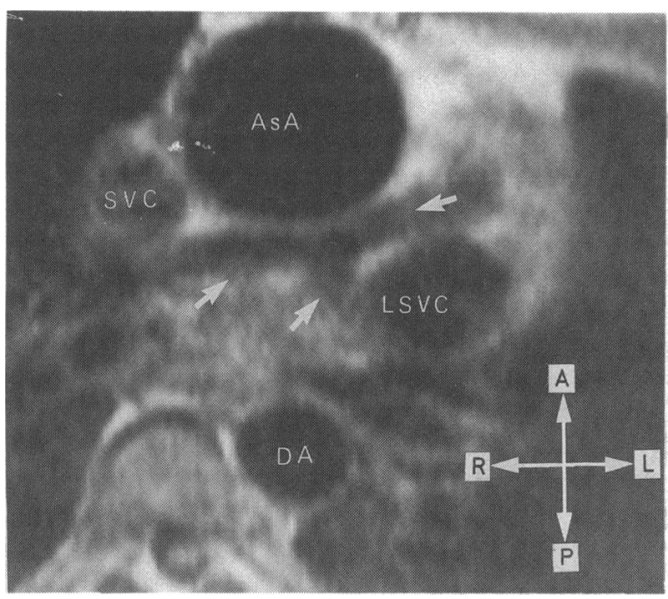

Fig 2 Magnetic resonance image of transverse slice in patient 6 showing hypoplastic central pulmonary arteries (arrows). As A, ascending aorta; SVC, superior vena cava; $L S V C$, left superior vena cava; $D A$, descending aorta. the $R$ wave. The magnetic resonance images were interpreted without knowledge of the other findings and were subsequently compared with the most recent angiographic and surgical data.

\section{Results}

The table summarises the results. Confluent pulmonary arteries were seen on the magnetic resonance images in six patients (figs 1 and 2). They could be readily identified from their shape and position in the mediastinum. In five the picture accorded with the angiographic and surgical findings. In the sixth (patient 5) no central pulmonary arteries were seen at angiography or surgery, but they could have been missed owing to their small size.

A total of 15 collateral origins from the descending aorta could be identified on the magnetic resonance images compared with 18 on angiography (figs 3 and 4). Two were missed in patient 10 , but the images were technically inadequate. It was possible to fol-

\section{irgery}

\section{ite}

Findings

Procedure

RPA present

LPA $5 \mathrm{~mm}$

$3 \mathrm{~mm}$ right hilar vessel

Confluent PAs,

984 LPA $3 \mathrm{~mm}$, RPA $2 \mathrm{~mm}$

963

RPA present

LPA $5 \mathrm{~mm}, 2$ collaterals to left
RSA-collateral shunt

Attempted Ao-RPA shunt Duramater Ao-LPA shunt Exploration LPA

Ao-PT direct shunt

Left Ao-collateral shunt

Left Blalock shunt

Right Blalock shunt

Left Blalock shunt

Right Ao-collateral shunt

Ao-confluence shunt

Left Blalock shunt
Left Blalock shunt
Small LPA, no RPA

No collaterals

No shunt seen

Confluent PAs

2 collaterals to left

1 collateral to right and left

Ao-PA shunt patent

Confluent PAs

1 collateral to right

1 collateral to left

No shunt seen

Large LPA: small RPA

No collaterals

Patent shunt

Confluent PAs

1 collateral to right

No shunt seen

Confluent PAs

1 collateral (?) side

Patent shunts

No PAs seen

3 collaterals to left, 1 collateral to right

Patent shunt

Confluent PAs

2 collaterals to left

1 collateral to right

No shunt seen

Confluent PAs. No collaterals

No shunt seen

Non-confluent PAs

1 collateral to left 


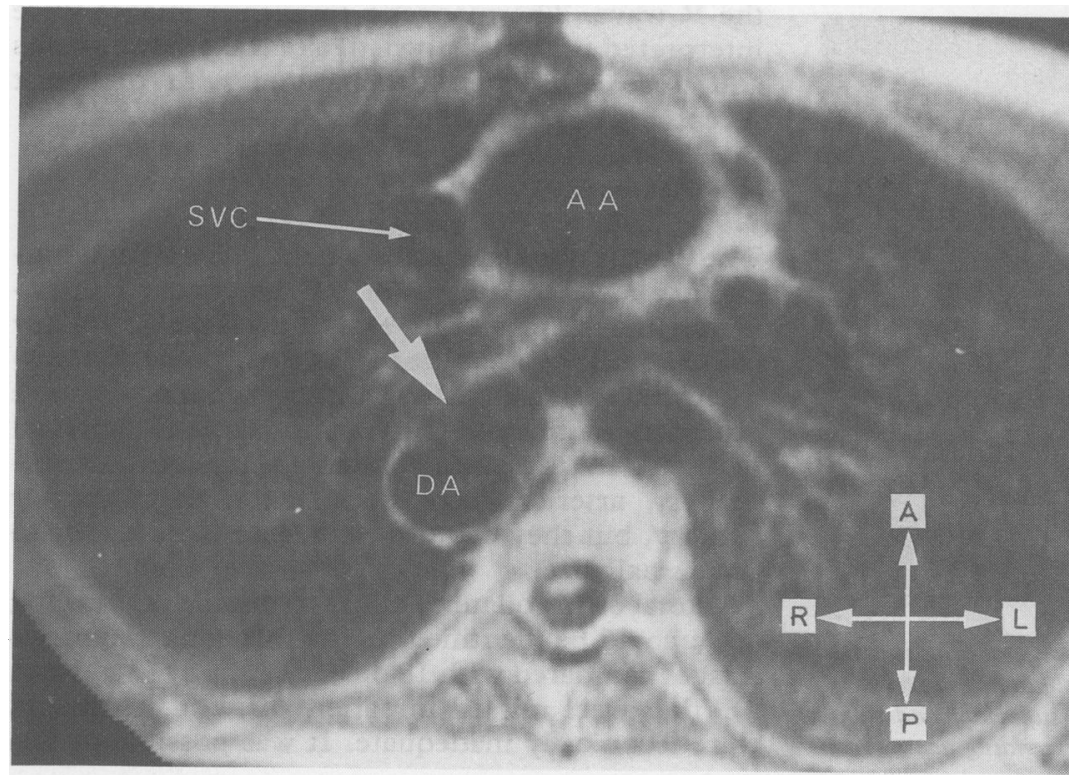

Fig 3 Magnetic resonance image of transverse slice in patient 8 showing origin of systemicpulmonary collateral (arrow) from descending aorta which is of a similar diameter. The vessel has a stenosis and passes to the left hilum. $S V C$, superior vena cava; $A A$, ascending aorta; $D A$, descending aorta.

low the course of most of the collaterals to the hilum, but not possible to establish their distal connections and interconnections. This is in contrast with selective angiography where such information can usually be obtained.

The magnetic resonance images and angiographic and surgical findings showed that five of the 11 surgical shunts were patent and two were blocked (figs 5 and 6). The state of the other four shunts on the angiograms was uncertain, but as they were not seen on the magnetic resonance images they were assumed to be occluded.

\section{Discussion}

Many patients with pulmonary atresia who survive into adult life have undergone multiple investigations and operations, like the ten patients in this study. At catheterisation there are practical problems in providing a complete angiographic assessment. Aortic flow is usually high so that aortography alone is inadequate. Selective catheterisation is necessary and failure to intubate all the branches is common, so that even in the first instance more than one investigation may be required to demonstrate the complete anatomy. ${ }^{6}$ The vital information is the size and connections of the central pulmonary arteries, which may be difficult to see on angiography as they are rarely connected directly to the aorta and fill only by retrograde flow. ${ }^{7}$ In some instances pulmonary venous wedge angiography may be necessary to demonstrate them. ${ }^{8}$ These vessels could be easily identified on the magnetic resonance images from their resem $\vec{\vartheta}$ blance to a seagull in flight and their position in the mediastinum. ${ }^{3}$

In contrast, the number of origins of collaterap arteries arising from the descending aorta were underestimated on the magnetic resonance images? this may be because it is difficult to be certain abou? the number of origins from the aorta on selective angiography, as an early bifurcation may lead to an overestimation. Beyond the origin of these vessels, $i \stackrel{3}{3}$

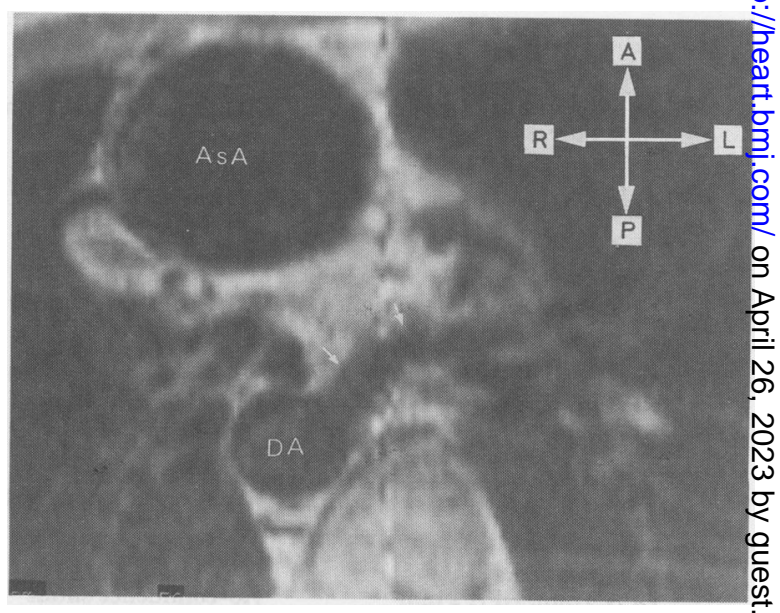

Fig 4 Magnetic resonance image of transverse slice in patient 3 showing origin of systemic-pulmonary collateral (arrows) from descending aorta (DA) passing to left hilum. $A s A$, ascending aorta. 


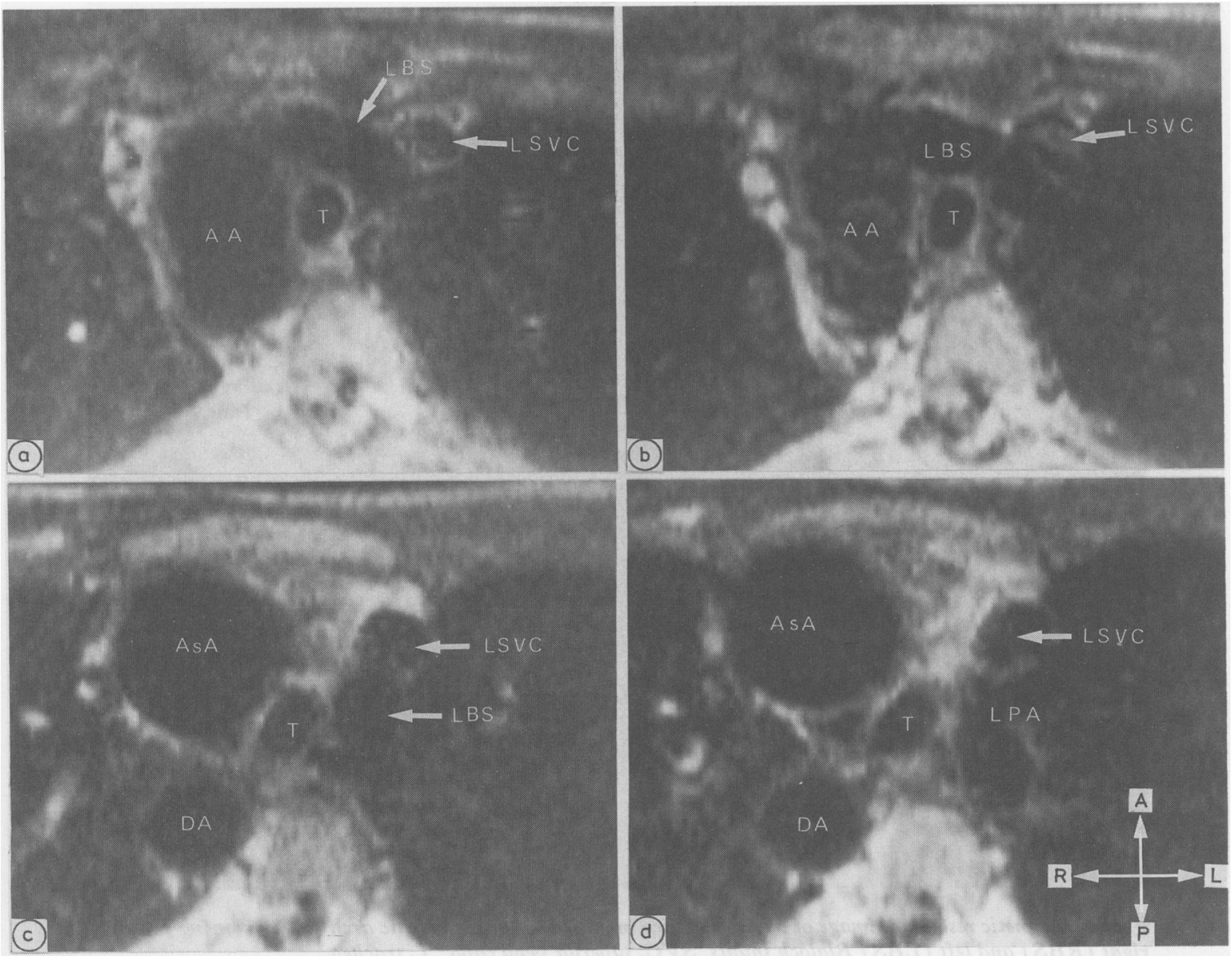

Fig 5 Magnetic resonance images of contiguous transverse slices ( $a-d)$ in patient 5 showing widely patent left Blalock shunt (LBS). Slice $a$ is cranial at the level of the aortic arch and slice $d$ caudal through the hilar region. AA, aortic arch; AsA, ascending aorta; DA, descending aorta; $L S V C$, left superior vena cava; $T$, trachea; $L P A$, left pulmonary artery.

was possible in most instances to follow them to the hilum but not possible to establish their distal vascular connections. This information is of importance when planning operation, as the number of segments in the lungs to which the true pulmonary arteries, as opposed to collateral arteries only, are connected is an important determinant of prognosis. ${ }^{9-11}$ Selective angiography remains the only method of demonstrating such connections and therefore will still be required for this purpose.

There was good agreement between the magnetic resonance and the angiographic and surgical findings on the state of surgical shunts. In those four patients in whom the state of the shunt was uncertain from the angiographic data, failure to visualise a patent shunt by magnetic resonance was an indication that the shunt was likely to be occluded.

Magnetic resonance imaging in pulmonary atresia provides an alternative non-invasive method of obtaining much of the anatomical information required to plan surgical treatment and should reduce the need for invasive angiography in these patients.

\section{References}

1 Jefferson K, Rees S, Somerville J. Systemic arterial supply to the lungs in pulmonary atresia and its relation to pulmonary artery development. $\mathrm{Br}$ Heart $J$ 1972;34:418-27.

2 Somerville J. Management of pulmonary atresia. $\mathrm{Br}$ 


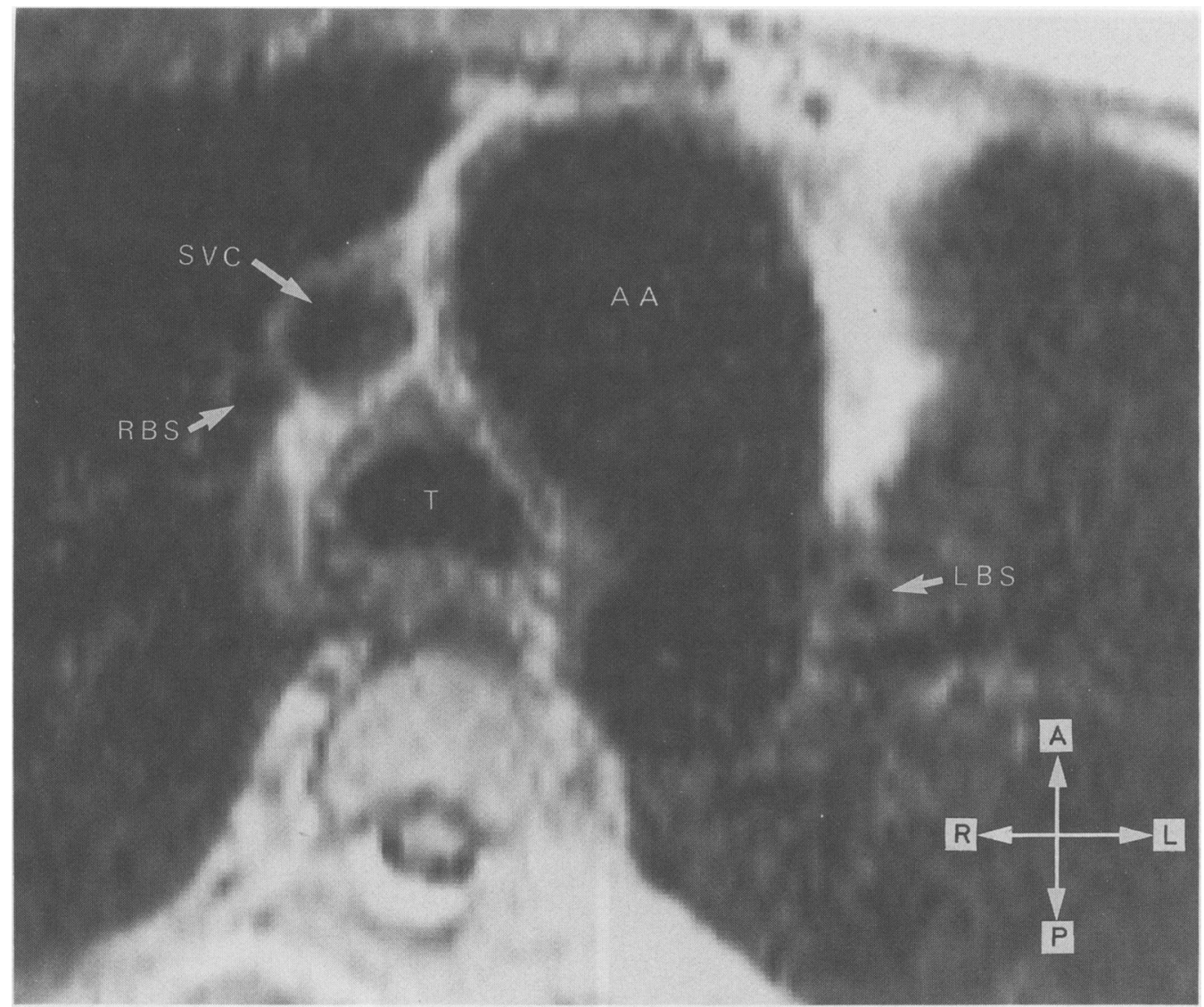

Fig 6 Magnetic resonance image of transverse slice in patient 6 through aortic arch (AA) showing patent right (RBS) and left (LBS) Blalock shunts. SVC, superior vena cava: $T$, trachea.

Heart $J$ 1970;32:641-51.

3 Somerville J, Saravalli O, Ross D. Complex pulmonary atresia with congenital systemic collaterals: classification and management. Arch Mal Coeur 1978;71:322-8.

4 Chesler E, Beck W, Shrire V. Selective catheterization of pulmonary or bronchial arteries in the preoperative assessment of pseudo-truncus arteriosus type IV. Am J Cardiol 1970;26:20-4.

5 Jacobstein MD, Fletcher BD, Nelson AD, et al. Magnetic resonance imaging: evaluation of palliative systemic-pulmonary shunts. Circulation 1984;70: 650-6.

6 Davis GD, Fulton RE, Ritter DG, et al. Congenital pulmonary atresia with ventricular septal defect: angiographic and surgical correlates. Radiology 1978; 128:133-44.

7 Fulton RE, Davis GD. Congenital pulmonary atresia: photographic subtraction as an aid in recognizing hypoplastic pulmonary arteries. AJR 1978

$$
\text { 131:1003-7. }
$$

8 Nihill MR, Mullins CE, McNamara DG. Visualizatioṇ. of pulmonary arteries in pseudotruncus by pul $\delta$ monary vein wedge angiography. Circulation 1978;58:140-7.

9 Fäller K, Haworth SG, Taylor JFN, Macartney FJ? Duplicate sources of pulmonary blood supply in pul? monary atresia with ventricular septal defect. $B$ z Heart $J$ 1981;46:263-8.

10 Haworth SG, Macartney FJ. Growth and development of pulmonary circulation in pulmonary atresia witis ventricular septal defect and major aortopulmonar $\hat{\omega}$ collateral arteries. Br Heart $J$ 1980;44:14-24.

11 Pacifico AD, Allen RH, Colvin EV. Direct reconstruction of pulmonary artery arborization anomal and intracardiac repair of pulmonary atresia withe ventricular septal defect. Am J Cardiol 1985;55: 1647-9. 